\title{
Editorial
}

\section{Gut aufpassen!}

Talsperren sind oft sehr groß. Sie sind elementare Bestandteile unserer Kulturlandschaft geworden. Sie sind heute da und werden auch morgen da sein. Eigentlich ist gar nicht abzusehen, dass diese Bauwerke jemals wieder niedergerissen werden. Das würde ja meist auch gar nicht funktionieren, da sich die Flusssysteme nach dem Bau der Talsperren stark verändern. Material lagert sich in den neuen Seen ab. Die ökologischen Zusammenhänge werden neu durchgemischt.

Die Talsperren bleiben also uns und den nachfolgenden Generationen erst einmal erhalten, womit es gilt, richtig umzugehen.

Die Seen der Talsperren sind ja der eigentliche Zweck des Ganzen. Sie stellen Brauchwasser zur Verfügung, sie schützen uns vor Hochwasser und sie speichern sehr viel Energie. Diese Energie wird in Österreich in bester Weise zur bedarfsgerechten Erzeugung von Wasserkraft genutzt. Sollte jedoch eine Talsperre einen ernsthaften Schaden erleiden - dann würde genau diese Energie eine Katastrophe in den flussab gelegenen Talräumen verursachen. Das müssen wir vermeiden. Dies ist die gemeinsame Verantwortung der Betreiber, der zuständigen öffentlichen Einrichtungen und der Fachleute in Österreich.

Österreich ist hier gut aufgestellt. Mit der Staubeckenkommission kümmert sich ein Expertengremium in einer sehr persönlichen, engagierten und großteils ehrenamtlichen Form um die Sicherheit der großen Stauanlagen. Jede größere Talsperre wird damit im Laufe der Zeit jedem Kommissionsmitglied mehr oder weniger vertraut. Genau dadurch gelingt es, einen hohen und doch ziemlich gleichmäßigen Sicherheitsstandard in ganz Österreich zu halten. Dieses in hohem Maße personengebundene System ist weltweit einzigartig.

In Deutschland läuft dies alleine schon aufgrund der Größe des Landes anders. Dort wird versucht, das einheitliche Sicherheitsniveau der Stauanlagen mit einem deutlich größeren Aufwand an Papier festzuschreiben. DIN-Normen und ergänzende Merkblätter beschreiben den Stand der Technik.

Die Stauanlagen werden älter. Ihre Zahl nimmt weiter zu. Das bedeutet, gerade bei der regelmäßigen Überprüfung der Sicherheit, einen doch erheblichen Zeitaufwand. Hier benötigt die Österreichische Staubeckenkommission nun die Unterstützung der Bundesländer. Hierfür werden gerade die Grundlagen erstellt.

Natürlich werden unsere Talsperren ständig überwacht. Die Bauwerke werden im „normalen Leben“ täglich oder wöchentlich visuell kontrolliert. Verformungen werden gemessen, Sickerwasserabflüsse beobachtet und Verschlüsse getestet. Das ist elementar wichtig. Ab und zu müssen wir aber noch mehr machen und einen Schritt zurücktreten! Dann ist es wichtig, die Anlagen im Detail inspizieren zu lassen, die Bemessungsgrundlagen zu überprüfen, den Bezug zum aktuellen Stand der Technik zu bewerten und sich ein sehr eingehendes Bild über den Gesamtzustand der Anlage zu machen. Als Ergebnis dieser tiefergehenden Überprüfungen im Abstand von typischerweise 5 Jahren in Österreich und 10 Jahren in Deutschland steht dann eine Aufstellung von möglichen Anpassungserfordernissen und eine grundsätzliche Bewertung der Anlagensicherheit.

Die Namen und die konkreten Ausgestaltungen dieser besonderen Sicherheits-Checks variieren. In Österreich sind hier die von der öffentlichen Verwaltung bestellten Fachleute federführend. In Deutschland liegt die Verantwortung für die Organisation der „Vertieften Überprüfungen“ alleine in der Hand der Betreiber. Die Aufsichtsbehörde wird regelmäßig informiert und kontrolliert insbesondere die Ergebnisse dieser Untersuchungen. 
Wichtig ist, dass wir auf unsere Talsperren aufpassen! Durch unterschiedliche zeitliche, organisatorische und inhaltliche Ebenen kann ein sehr hohes Sicherheitsniveau über Generationen gehalten werden. Auch wenn es uns manchmal schwerfällt, sollten wir dabei immer wieder neue Köpfe und neue Ideen in diese wichtigen Arbeiten einbinden. Nur so kann es gelingen, ein wirklich gutes und umfassendes Bild der Sicherheit der großen Staubauwerke über lange Zeiten zu halten. Dies ist der richtige Weg!

\section{Interessenkonflikt}

M. Aufleger gibt an, dass kein Interessenkonflikt besteht.

Univ.-Prof. Dr.-Ing. M. Aufleger ( $₫)$

Arbeitsbereich Wasserbau, Institut für

Infrastruktur, Universität Innsbruck,

Innsbruck, Österreich

markus.aufleger@uibk.ac.at 